\title{
Elejalde Syndrome: The Silvery Hair Syndrome
}

\author{
Fehmida Najmuddin ${ }^{1 *}$, Rajesh Rai $^{1}$, Keya Lahiri ${ }^{1}$, Priya Patil Cholera ${ }^{2}$ \\ ${ }^{1}$ Department of Paediatrics, D Y Patil medical college, Hospital \& research centre, India \\ ${ }^{2}$ Department of Ophthalmology, D.Y. Patil medical college, Hospital \& research centre, India
}

Received: June 01, 2015; Accepted: August 04, 2015; Published: September 08, 2015

*Corresponding author: Dr. Fehmida Najmuddin, Department of paediatrics, D Y Patil Medical College, Hospital \& Research Centre, Maharashtra, India, Tel: 0091-9920030533; e-mail:fehmidanc@hotmail.com

\begin{abstract}
Elejalde Syndrome is a rare autosomal recessive disorder. The characteristic features are silvery hair and profound central nervous system dysfunction. Unevenly distributed melanin granules are observed on hair analysis. We hereby describe, a two and half year old female child with neuroregression, diffuse hypopigmentation and silvery hair who was diagnosed to have this syndrome.
\end{abstract}

Keywords: Silvery hair; Elejalde Syndrome; Neuroregression

\section{Introduction}

Elejalde Syndrome (ES) is also known as neuro-ectodermal melanolysosomal disease [1]. It forms a spectrum of the silvery hair syndromes. This disorder is characterized by silvery hair, pigment and neurological abnormalities like seizures, hypotonia and intellectual impairment. The immunological function is usually preserved in this syndrome. These patients have hypopigmentation due to impaired melanosome transport [2].

\section{Case Report}

A two and half year old female child, from India presented to our tertiary care hospital with complaints of inability to walk since past 6 months. Child was apparently normal till the age of 2 years and had achieved all milestones for her age.

The mother noticed progressive weakness of the lower limbs (right more than left limb) initially which progressed to involve the trunk muscles and later the upper limbs. The progressive weakness resulted in regression of milestones. There was no history of fever, rash, recent intramuscular injection, convulsions, altered sensorium, involuntary movements, cranial nerve involvement, trauma or diarrhoea. The child had no significant illnesses or admissions in the past. Child was born of a third degree consanguineous marriage and had a healthy 7 year old sibling. She was born full term with birth weight of $3000 \mathrm{gms}$. Antenatal and postnatal history was insignificant. Child was partially immunised and belonged to a lower middle social class.

Vital parameters were stable with Grade-1 protein energy malnutrition and pallor. There was silvery hair observed on the scalp, eyebrows, eyelid lashes, back and limbs. A hypopigmented macule was observed over left lower eyelid measuring $1 \times 1.5$ $\mathrm{cm}$ (Figure 1). Multiple hypopigmented lesions measuring $0.5 \mathrm{x}$
$0.5 \mathrm{~cm}$ over both the arms and thigh and an epidermal nevus measuring $7 \times 1 \mathrm{~cm}$ was seen. There was dental caries along with a sacral dimple.

Higher mental functions and cranial nerve examination was normal. On motor system examination, tone was bilaterally reduced in all the four limbs with power more than $3 / 5$ and reflexes being normal. There were no signs of meningeal irritation, cerebellar or sensory system involvement. There was hepatosplenomegaly with rest of the systemic examination being normal. On the basis of silvery hair and neuroregression a diagnosis of silvery hair syndrome was made.

Haemoglobin was $12.2 \mathrm{gm} / \mathrm{dl}$ with total leucocyte count of $10,900 / \mathrm{cmm}$ and platelets of 2,50,000/cmm. Peripheral smear examination was normal with no intracytoplasmic granules seen in the neutrophils. Random blood sugar was $124.3 \mathrm{mg} / \mathrm{dl}$ with normal serum electrolytes, liver function tests, prothrombin time, partial thromboplastin time and cerebrospinal fluid analysis. Immunological studies in the form of serum immunoglobulin levels and lymphocyte subset analysis was normal. Magnetic Resonance Imaging (MRI) of the brain showed cerebellar atrophy with no basal ganglia calcification while the MRI of the spinal cord was normal. Hair analysis revealed alternate pigmentation and large uneven clusters of pigment suggestive of silvery hair

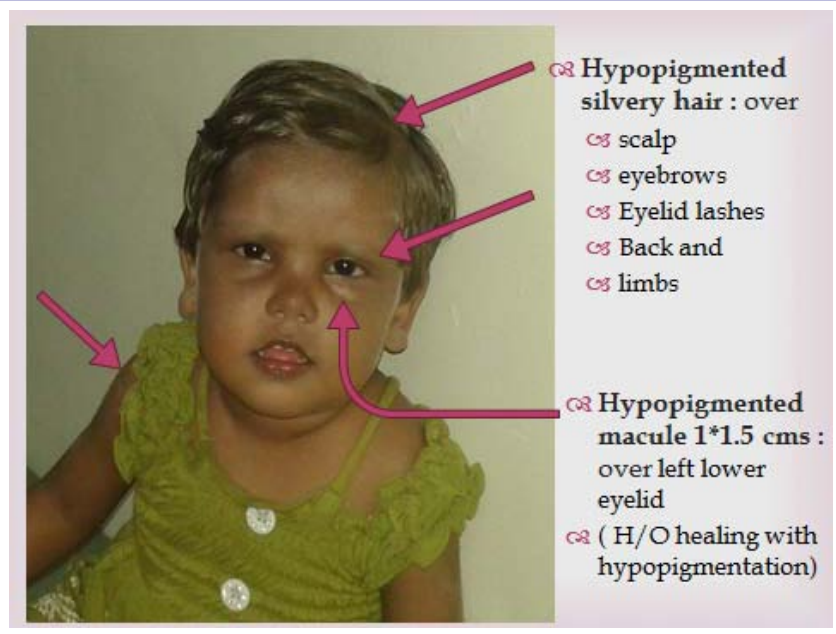

Figure 1: Showing features of Elejalde syndrome. 
syndrome (Figure 2). Ophthalmology examination did not reveal any abnormality.

The features of silvery hair, cerebellar atrophy, hypopigmented skin lesions, and absence of recurrent infections was suggestive of Elejalde Syndrome.

\section{Discussion}

ES is a rare syndrome, which was first described by Elejalde et al. [3]. Only few cases have been reported so far. It is an autosomal recessive disorder which is characterized by silvery hair, neuroregression and normal immunological profile [1] The molecular basis of this syndrome is still not understood. Hair analysis in these patients shows characteristic small and large clumps of melanin which are irregularly distributed [4] Maturation defect results in incomplete melanosome transport leading to hypopigmentation. The most common presentation of ES is neurological manifestation in the form of seizures, hypotonia, hemiplegia, quadriplegia, ataxia, and ocular abnormalities [5]. The onset of central nervous system involvement varies from 1 month to 11 years. The second most common feature is pigmentary abnormalities.

ES was earlier considered as an allelic variant of Griscelli Syndrome (GS), therefore it has also been referred to as GS Type 1 [6]. In 2003, Bahadoran et al. [7] reported that, though there was hair shaft melanin clumping seen in ES patients which is similar to GS, there was an abnormal melanin maturation noted.

Recently, studies have shown that ES is to be considered same as GS Type 1. Apart from silvery hair and hypopigmentation, there is severe, early onset central nervous system dysfunction [8.9]

In literature, 3 forms of GS have been reported. Type 1 GS is caused by mutations in MYO5A and is associated with severe neurological impairment. Type 2 GS is caused by mutations in GTPase RAB27A and is associated with immunodeficiency. Type $3 \mathrm{GS}$ is caused by gene mutations involving melanophilin (Mlph) and is associated with hypopigmentation $[10,11]$

The closest differential diagnoses of ES are Griscelli syndrome

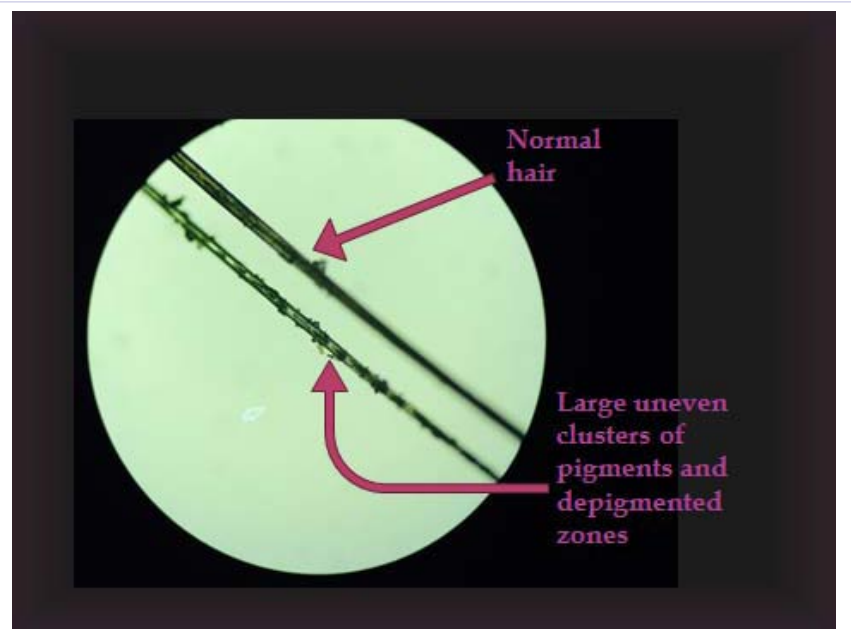

Figure 2: Hair analysis showing features suggestive of Elejalde Syndrome.
(GS) and Chediak- Higashi Syndrome (CHS). Both GS and CHS are rare, autosomal disorder's which present during infancy and early childhood, but they have recurrent infections due to affection of humoral and cell mediated immunity. In addition to this, CHS is associated with impaired neutrophil chemotaxis and presence of abnormal giant intra-cytoplasmic granules in neutrophils found in the peripheral blood smear or bone marrow aspiration.

Management of these patients is usually symptomatic and they should be closely monitored for follow up when they start deteriorating neurologically. Usually the prognosis is fatal and mortality is due to central nervous system involvement and its complications. In 2001, Ivanovich et al. [1] reported the oldest living child of ES in literature. To conclude, any patient presenting with silvery hair and neurological involvement should be investigated for silvery hair syndrome i.e., Elejalde syndrome, Griscelli Syndrome and Chediak -Higashi Syndrome.

\section{References}

1. Ivanovich J, Mallory S, Storer T, Ciske D, Hing A. 12-year-old male with Elejalde Syndrome( neuroectodermal melanolysosomal disease). Am J Med Genet. 2001; 98(4):313-6.

2. Lambert J, Vancoillie G, Naeyaert JM. Elejalde Syndrome revisited. Arch Dermatol. 2000; 136(1):120-1.

3. Elejalde BR, Holguin J, Valencia A, Gilbert EF, Molina J, Marin G, et al. Mutations affecting pigmentation in man: I. Neuroectodermal melanolysosomal disease. Am J Med Genet. 1979; 3(1):65-80.

4. Duran-McKinster C, Rodriguez-Jurado R, Ridaura C, de la Luz Orozco-Covarrubias M, Tamayo L, Ruiz-Maldonando R. Elejalde Syndrome--a melanolysosomal neurocutaneous syndrome: clinical and morphological findings in 7 patients. Arch Dermatol. 1999; 135(2):182-6.

5. Cahali JB, Fernandez SA, Oliveira ZN, Machado MC, Valente NS, Sotto MN. Elejalde syndrome: Report of a case and review of the literature. Pediatr Dermatol. 2004; 21(4):479-82.

6. Sanal O, Yel L, Kucukali T, Gilbert-Barnes E, Tardieu M, Texcam I, etal. An allelic variant of Griscelli disease: presentation with severe hypotonia, mental motor retardation and hypopigmentation consistent with Elejalde syndrome ( neuroectodermal melanolysosomal disorder). J Neurol. 2000; 247(7):570-2.

7. Bahadoran P, Ortonne JP, Ballotti R, de Saint-Basile G. Comment on Elejalde syndrome and relationship with Griscelli syndrome. Am J Med Genet A. 2003; 116A(4):408-9.

8. Reddy RR, Babu BM, Venkateshwaramma B, Hymavathi Ch. Silvery hair syndrome in two cousins: Chediak-Higashi syndrome vs Griscelli syndrome, with rare associations. Int J Trichology. 2011; 3(2):107-11. doi: 10.4103/0974-7753.90825.

9. Valente NY, Machado MC, Boggio P, Alves AC, Bergonse FN, Casella $E$, et al. Polarized light microscopy of hair shafts aids in the differential diagnosis of Chédiak-Higashi and Griscelli-Prunieras syndromes. Clinics (Sao Paulo). 2006; 61(4):327-32.

10. Sahana M, Sacchidanand S, Hiremagalore R, Asha G. Silvery grey hair: Clue to diagnose immunodeficiency. Int J Trichology. 2012; 4(2):835. doi: 10.4103/0974-7753.96910.

11.Vincent LM, Gilbert F, DiPace JI, Ciccone C, Markello TC, Jeong A, et al. Novel $47.5-\mathrm{kb}$ deletion in RAB27A results in severe Griscelli Syndrome Type 2. Mol Genet Metab. 2010; 101(1):62-5. doi: 10.1016/j.ymgme.2010.05.015. 